\title{
A Atuação do Psicólogo em ONG/AIDS
}

Psychological practices at AIDS/NGOs

Emerson F. Rasera \&

Carmem Lucia

Graminha Issa

Universidade

Federal de Uberlândia

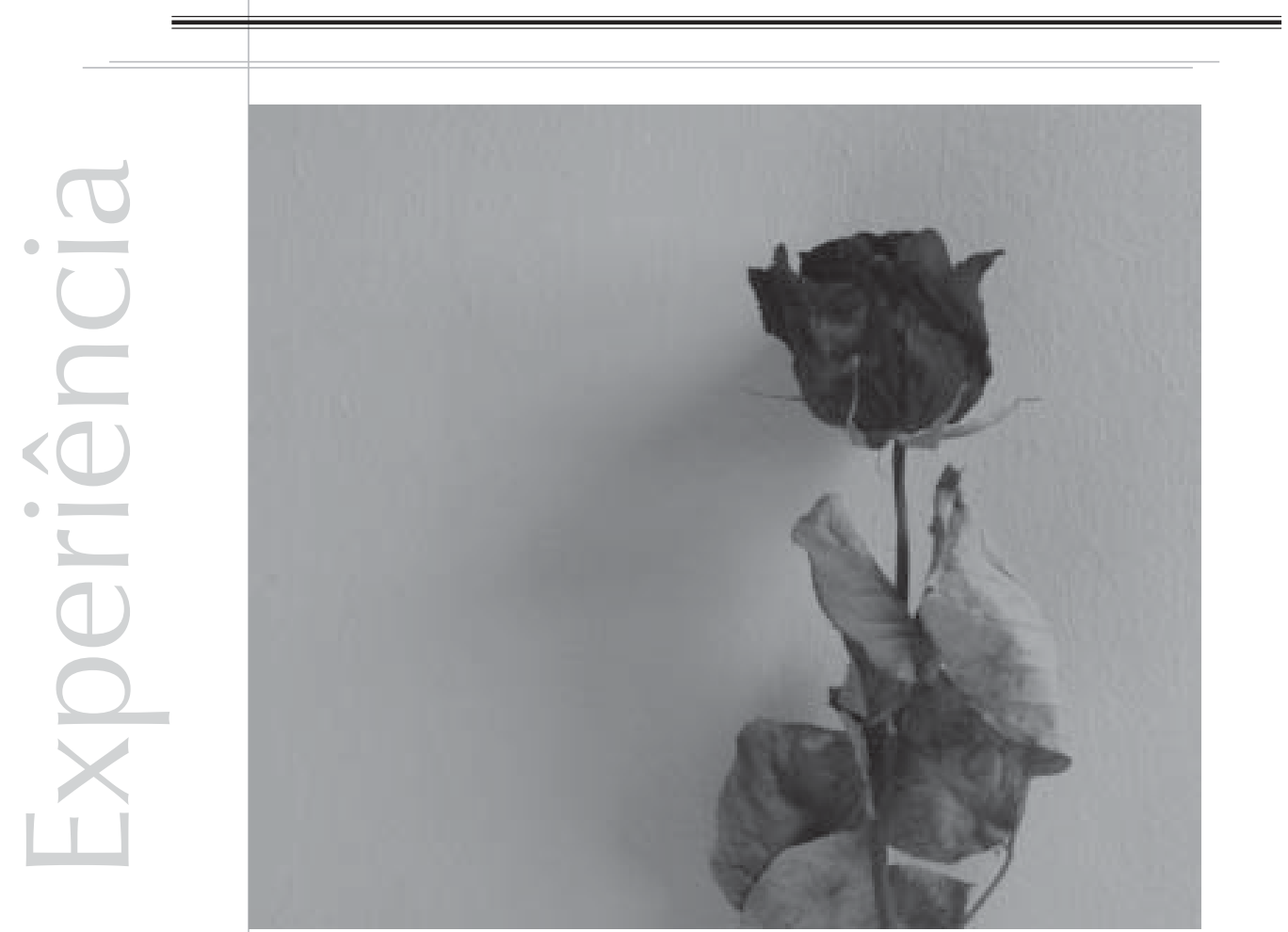


Resumo: Desde o início da epidemia, a Psicologia tem contribuído na luta contra a AIDS através da assistência psicológica aos portadores do HIV e seus familiares e na prevenção às DST/AIDS. Associado à epidemia e às respostas do movimento social organizado, surgiu um novo contexto de atuação para os profissionais da Psicologia: as organizações não-governamentais de luta contra a AIDS. Considerando a escassez de literatura brasileira sobre a contribuição da Psicologia nesse contexto, o objetivo deste relato de experiência é descrever a atuação dos psicólogos em uma organização não governamental de Ribeirão Preto/São Paulo/Brasil, o Grupo Humanitário de Incentivo à Vida (GHIV). A diversidade de atividades realizadas pelos psicólogos nessa ONG vão do atendimento psicoterápico individual e coordenação de diferentes tipos de práticas grupais à organização da estrutura da ONG e à supervisão da atuação política dos membros da mesma. Entre os desafios gerados pela atuação do psicólogo nesse contexto estão a integração entre prática profissional e compromisso social e o lugar da pessoa portadora na relação com o psicólogo. Eles explicitam como a AIDS veio questionar práticas profissionais tradicionais e exigir alternativas na atenção em saúde.

Palavras-chave: Psicologia social da saúde, ONG, atuação profissional, HIV/AIDS.

\begin{abstract}
Since the beginning of the epidemic, Psychology has contributed in the fight against AIDS through psychological care for those living with AIDS, their relatives and STD/AIDS prevention. Related to the AIDS social movement, a new context of work for psychologists emerged: the AIDS non-governmental organizations. Considering the scarcity of Brazilian literature about the contribution of Psychology in this context, the objective of this article is to describe the psychological practices developed at an AIDS non-governmental organization in Ribeirão Preto/São Paulo/Brazil, the Grupo Humanitário de Incentivo à Vida. The diversity of activities performed by psychologists in this NGO goes from individual psychotherapy and coordination of different types of group to the organization of the structure of the NGO and the supervision of its political actions. Among the challenges faced by the psychologist in this context are the integration between professional practice and social activism and the relationship between the HIV carrier and the psychologist. They point out how AIDS challenges traditional professional practices and demands changes in healthcare.
\end{abstract}

Key words: Health Social Psychology, NGO, professional practice, HIV/AIDS.

Desde o surgimento da epidemia, a Psicologia tem contribuído de diferentes formas na luta contra a AIDS, tanto na produção de conhecimento que subsidia ações nesse campo, na assistência psicológica aos portadores do HIV e a seus familiares como na prevenção às DST/AIDS. A vasta literatura a esse respeito aponta o fato de, entre as diversas ações realizadas pelos psicólogos, estarem o aconselhamento pré-pós testes, a psicoterapia individual a pacientes ambulatoriais e internados, a psicoterapia de grupo com pacientes soropositivos e com seus familiares bem como com os profissionais que lidam com pessoas com HIV/AIDS, além das intervenções de prevenção de diferentes tipos junto a variados grupos populacionais (Zegans et al., 1994, Catalan, 1995; IDAC/BANCO DE HORAS, 2000; Buchalla, Paiva, 2002; Silva et al., 2002; Saldanha, Figueiredo, Coutinho, 2004).

Associado à epidemia e às respostas do movimento social organizado, surgiu um novo contexto de atuação para os profissionais da Psicologia: as organizações não-governamentais de luta contra a AIDS. Essas organizações nãogovernamentais (ONGs) têm caracterizado uma forma do enfrentamento dessa epidemia baseada no caráter associacionista e coletivo das respostas sociais que extrapolam os contextos médico-sanitários tradicionais. No 
Brasil, elas surgem em meados da década de 80, e se expandem até o número de quinhentas, no ano 2000, conforme o Cadastro do Ministério da Saúde. Representadas por instituições que vão de sindicatos a igrejas bem como aquelas criadas especificamente para a luta contra a AIDS, essas ONGs realizam atividades de apoio e assistência a pessoas portadoras e seus familiares, prevenção e educação em DST/AIDS e articulação política na luta pelos direitos das pessoas soropositivas. São iniciativas, muitas vezes, marcadas pela participação da população soropositiva e pela criação de fortes laços identitários e de solidariedade.

Apesar da literatura sobre movimentos sociais, decorrente especialmente de estudos no campo da Sociologia e das Ciências Políticas, ao se analisar as características dessas ONGs (Câmara, Lima, 2000; Câmara, 2002; Silva, 2002), as relações que se estabelecem em seu interior, bem como com outros atores sociais, verifica-se que ainda há uma escassez de literatura brasileira sobre a contribuição da Psicologia no contexto das ONGs/AIDS.

Além disso, os desafios trazidos pela AIDS no campo da Psicologia, especialmente da Psicologia da saúde, deixaram expostas as fragilidades da atuação profissional no contexto das ONGs/AIDS. A Psicologia brasileira foi historicamente marcada por um viés individualista, focada na assistência em saúde e voltada para o atendimento da população das camadas privilegiadas. Dessa forma, os psicólogos não estavam preparados para a atuação junto à população portadora do HIV em instituições com forte compromisso político, através de práticas que implicassem uma redefinição das relações entre Psicologia, saúde e sociedade. Constitui-se, ainda, um desafio ressocializar a Psicologia da saúde para que tais fragilidades possam ser devidamente compreendidas e transformadas (Dimenstein, 1998, 2001; Spink, 2003).

Assim, considerando a relevância desse tema e a escassez de literatura sobre o mesmo, este relato de experiência tem como objetivo descrever a participação do psicólogo na criação e na estruturação de uma organização não-governamental de luta contra a AIDS. Buscaremos apresentar um breve histórico do processo de criação da ONG, os programas realizados e a participação do psicólogo em cada um deles. Finalizaremos o artigo refletindo sobre algumas questões trazidas por essa história de participação da Psicologia nesse contexto específico.

\section{GHIV: o processo de criação da ONG}

O Grupo Humanitário de Incentivo à Vida (GHIV) é uma organização não governamental, sem fins lucrativos, cuja missão é promover melhor qualidade de vida para as pessoas portadoras do HIV/AIDS em Ribeirão Preto/ SP. Seu início informal, em 1993, consistiu de um grupo de auto-ajuda coordenado semanalmente por uma pessoa portadora do HIV, nas dependências de um sindicato no centro da cidade de Ribeirão Preto. Frente a diversas dificuldades enfrentadas pelo seu coordenador, esse trabalho foi interrompido, sendo retomado em 1995, quando fez uma parceria com um psicólogo voluntário, que passou a co-coordenar o grupo. Nesse momento, o papel do psicólogo foi o de promotor das potencialidades de autoorganização e cuidado mútuo de pessoas que viviam condições de vida semelhantes. Ele servia como apoio e referência para as dificuldades trazidas pelo co-coordenador na relação com os participantes. A participação de uma psicóloga, em 1996, permitiu oferecer também um grupo terapêutico fechado, de final aberto, para pessoas portadoras.

A partir desses atendimentos em grupo, as pessoas portadoras buscaram discutir as necessidades dos serviços de saúde locais, participar de encontros nacionais de pessoas que viviam com HIV/AIDS e manifestar o interesse em formar uma ONG. O psicólogo 
passou então a atuar como estimulador para que buscassem informações sobre as exigências legais a fim de constituir uma organização, e definissem qual seria o perfil e os objetivos da ONG a ser criada. Acompanhando esse processo, pôde contribuir, através do diálogo e do questionamento crítico, para a reflexão sobre as decisões tomadas e o manejo das tensões criadas nessas negociações.

Os atendimentos terapêuticos não objetivavam criar uma ONG. Ela foi resultado de um processo de abertura às necessidades identificadas na relação com os participantes do grupo. A atuação do psicólogo foi pautada por uma sensibilidade a outras respostas além daquelas "psicologizadas" e individuais. Assim, não se entendia o que era relatado pelos participantes nos atendimentos psicológicos como referência a uma subjetividade isolada das condições socio-históricas de sua produção. Havia abertura na escuta do psicólogo para processos de coletivização que gerassem respostas políticas e organizacionais. Tal escuta implicava a legitimação concreta dessas respostas como uma forma de cuidado frente ao sofrimento psicológico presente no discurso dos portadores do HIV.

Ao mesmo tempo em que se articularam para a criação formal de uma organização não governamental, realizaram, com outras ONGs da cidade, o I Encontro Regional de Pessoas Vivendo com HIV/AIDS. O reconhecimento da qualidade desse trabalho culminou no recebimento do IV Prêmio Theo, oferecido pelo NEPAIDS/USP, em dezembro de 2001, à iniciativa de maior destaque no Estado de São Paulo na luta pelos direitos das pessoas portadoras. Em junho de 2002, foi fundado, formalmente, o GHIV.

No primeiro ano de atividade do GHIV, foi possível contabilizar mais de oitocentos atendimentos a cerca de duzentas pessoas portadoras e seus familiares, além de quarenta e uma reuniões de articulação entre ONGs/ AIDS, cuja amplitude teve um impacto indireto em mais de duas mil pessoas soropositivas na cidade de Ribeirão Preto e região. Os voluntários totalizavam vinte e oito profissionais, a maior parte deles psicólogos. Parcerias com o Programa de Atendimento Psicossocial à AIDS/FFCLRP, da Universidade de São Paulo, e com o Programa Municipal de DST/AIDS, de Ribeirão Preto, além do apoio permanente do SINSPREV - Sindicato dos Trabalhadores em Saúde e Previdência no Estado de São Paulo, facilitaram o desenvolvimento das atividades.

\section{A atuação do psicólogo no GHIV: suas ações, conceitos e valores}

A atuação do psicólogo no GHIV contemplava uma diversidade de ações, influenciadas por diferentes inspirações conceituais e valores. Entre as ações realizadas pelo psicólogo, estavam: 1) estudo e proposição da estrutura organizacional e seu funcionamento; 2) pronto atendimento psicológico; 3) psicoterapia individual; 4) psicoterapia de grupo; 5) grupos de apoio e grupos educativos; 6) promoção de redes de solidariedade; 7) treinamento de profissionais de saúde; 8) assessoria e planejamento de ações de defesa dos direitos dos portadores; 9) integração de equipes de trabalho. Após a formalização do GHIV e a definição de sua estrutura de funcionamento, as ações desenvolvidas pelo psicólogo estavam associadas às propostas e aos objetivos de cada núcleo e programa da ONG.

Inicialmente, no processo de fundação da ONG, o psicólogo pôde contribuir com uma proposta de estrutura organizacional (atuação 1). Nesse momento, o psicólogo, baseado nas experiências realizadas antes da formalização da ONG e dos objetivos formais assumidos por ela em seu estatuto e utilizando-se de seus conhecimentos sobre Psicologia organizacional,
A atuação do psicólogo foi pautada por uma sensibilidade a outras respostas além daquelas "psicologizadas" e individuais. 
Entre os principais conceitos e valores que orientovam as ações dos psicólogos desse Programa, estavam os de Psicologia cidadã e saúde integral. pôde, através de diversas reuniões com os membros da ONG, facilitar o processo de estruturação das atividades da instituição. Essa estruturação permitiu maior racionalidade no fluxo das atividades, clareza da divisão de responsabilidades e maior satisfação entre os voluntários. Tal proposta contemplava, além dos conselhos definidos estatutariamente, seis núcleos de atividades, quais sejam, o Núcleo de Atenção Psicossocial, de Cidadania Ativa, de Prevenção à AIDS e Combate à Discriminação, de Orientação Jurídica, Administrativo e de Educação Física.

Considerando os limites de espaço deste artigo e a centralidade desses núcleos para a discussão a ser realizada sobre a atuação do psicólogo, apresentaremos apenas as atividades dos primeiros dois núcleos, durante a primeira gestão da ONG, no período de junho de 2002 a junho de 2004.

O Núcleo de Atenção Psicossocial congregava três Programas, quais sejam, o Programa de Assistência Psicológica, o de Socialização e o de Adesão nos Ambulatórios. O Programa de Assistência Psicológica consistia numa oportunidade de atenção psicológica que buscava complementar a escassa oferta desse tipo de serviço na rede pública de saúde. Esse Programa tinha por objetivo oferecer atendimento especializado em saúde mental a todos aqueles que vivem com HIV/AIDS e contribuir para um melhor enfrentamento da doença e de suas implicações.

A principal ação do psicólogo nesse Programa consistia no oferecimento de diferentes modalidades de atenção psicológica (atuação 2, 3 e 4), descritas a seguir: I - plantão psicológico individual: buscava oferecer o pronto atendimento psicológico, triagem e encaminhamento para portadores e familiares; após agendamento, o usuário era atendido pelo psicólogo responsável pelo plantão; II grupo para portadores: oferecia apoio psicológico em grupo a pessoas portadoras do
HIV; ocorria semanalmente, tinha $1 \mathrm{~h} 30 \mathrm{~m}$ de duração e era co-coordenado por dois psicólogos. Os novos participantes do grupo eram encaminhados e preparados pelo plantão psicológico; III - atendimento psicológico individual: através de encaminhamentos do plantão psicológico, os usuários, segundo suas necessidades, tinham acesso ao atendimento psicológico individual. Tratava-se de um atendimento realizado nos consultórios de cada um dos psicólogos voluntários associados ao GHIV.

Entre os principais conceitos e valores que orientavam as ações dos psicólogos desse Programa, estavam os de Psicologia cidadã e saúde integral. Por meio do conceito de Psicologia cidadã, enfatizava-se que, em consonância com as novas posturas no campo psicológico, a ação do psicólogo buscava responder a um compromisso social. Dessa forma, a soropositividade era entendida como um fenômeno diretamente relacionado às desigualdades sociais, sejam econômicas, de gênero e/ou sexuais. A consideração dessas desigualdades na produção do sofrimento psíquico era fortemente enfatizada. Associada a essa visão, estava a de saúde integral, na qual as necessidades de saúde das pessoas portadoras compreendiam não só aspectos biológicos (tão enfatizados nos contextos de saúde dominados por um discurso biomédico) como também psicológicos e sociais. Por meio desse conceito, entende-se que a atenção em saúde mental tem implicações diretas para a qualidade de vida dos portadores ao promover melhores relações consigo próprios e com os outros ao seu redor.

O Programa de Socialização tinha por objetivo possibilitar uma alternativa de sociabilidade a pessoas portadoras do HIV visando a aumentar a rede de apoio e as oportunidades de lazer de seus participantes. Sua principal ação consistia na realização de encontros semanais de duas horas de duração, coordenados por dois psicólogos (atuação 5 e 6), cujo 
planejamento era previamente determinado com os participantes e então divulgado, a cada seis meses, nos ambulatórios especializados de saúde no atendimento da população soropositiva. Entre os diferentes tipos de atividades realizadas intercaladamente nesses encontros, estavam: I) vídeo e debate: após assistirem a um filme de ficção, os participantes conversam sobre o mesmo, o que promove o lazer e facilita a construção de novas amizades em um clima descontraído; II) "grupo de bate-papo": através de reuniões não estruturadas, os participantes traziam os assuntos que mais os afligiam e buscavam refletir em conjunto sobre tais dificuldades, de forma a facilitar seu enfrentamento. $\mathrm{O}$ apoio recebido e o compartilhar de informações e experiências aumentavam o vínculo entre os participantes; III) palestras informativas: contando sempre com a presença de um profissional convidado, os participantes recebiam novas informações e discutiam sobre diferentes aspectos do viver com HIV/AIDS, o que possibilitou o esclarecimento de suas dúvidas e a diminuição do sentimento de insegurança; IV) confraternização: realizada mensalmente, permitia aos participantes do GHIV a oportunidade de rever amigos e fortalecer coletivamente um senso de esperança.

Nesse Programa, cabia aos psicólogos promover a participação dos portadores na programação e no desenvolvimento das atividades bem como facilitar os processos grupais oriundos dessa relação entre os participantes e destes com a ONG. A atuação do psicólogo se baseava na valorização da integração dos portadores entre si. O vínculo com outros portadores para a realização de atividade de lazer levava ao combate de uma visão da AIDS como castigo mortal, o que diminuía o preconceito e favorecia a participação ativa da pessoa portadora na construção social de um novo jeito de viver com o HIV. Ao mesmo tempo, pautado pela busca de uma saúde integral, o psicólogo promovia oportunidades institucionais de socialização no combate ao isolamento, à depressão e ao sentimento de impotência, o que possibilitava uma forma de autocuidado com claras implicações para os outros aspectos do tratamento da pessoa portadora.

O Programa de Adesão em Ambulatório tinha como objetivo fortalecer iniciativas institucionais voltadas para a promoção da adesão da pessoa portadora do HIV ao tratamento e à vida, nos ambulatórios de DST/ AIDS do Município de Ribeirão Preto. As ações dos psicólogos (atuação 5, 6, 7 e 9) nesse Programa se dividiam em três tipos: I) grupo de adesão nos ambulatórios: realizados mensalmente junto aos profissionais e usuários de cada um dos cinco ambulatórios de DST/ AIDS de Ribeirão Preto: através de reuniões não estruturadas, buscava-se dar suporte às iniciativas de cada ambulatório na realização de grupos de adesão, que promoviam o acolhimento e a reflexão para os diversos atores e para as perspectivas envolvidas na questão da adesão, de forma a facilitar a construção conjunta de alternativas para as dificuldades aí enfrentadas; II) encontro dos grupos de adesão: realizado semestralmente com todos os participantes de cada um dos grupos de adesão da cidade, profissionais e usuários, com o objetivo de promover a troca de experiências entre os mesmos bem como valorizar a participação de todos; III) oficina temática: elaborada a partir das necessidades dos profissionais dos ambulatórios de DST/ AIDS, tinha o objetivo de sensibilizar, capacitar e integrar os profissionais para trabalharem com questões específicas no atendimento da pessoa portadora.

O papel do psicólogo no desenvolvimento dessas atividades foi orientado pelos seguintes conceitos e valores: A) adesão como fenômeno multifacetado: a adesão era entendida como a fusão dos aspectos biológicos, psicológicos e sociais que, de diferentes maneiras, influenciavam a participação da pessoa 
portadora em seu próprio tratamento, e somente a atenção cuidadosa a cada um desses aspectos permitiria compreender o processo de adesão; B) co-responsabilização entre profissionais e portadores do HIV: o tratamento era considerado como uma parceria entre dois tipos de especialistas: o profissional da saúde, de um lado, com seus conhecimentos científicos e a pessoa portadora, de outro lado, com o conhecimento sobre sua própria vida. A combinação desses conhecimentos propiciaria a criação das melhores alternativas de tratamento que levariam a melhor qualidade de vida tanto para as pessoas portadoras como para os profissionais da saúde; C) ênfase em atividades grupais: a promoção de atividades grupais favorecia a integração das pessoas portadoras, bem como da equipe profissional, gerando respostas coletivas sensíveis à realidade de cada ambulatório em suas particularidades e potencialidades.

O segundo núcleo a ser apresentado é o de Cidadania Ativa. Esse núcleo promovia a participação do GHIV na discussão e elaboração de políticas de saúde junto a orgãos governamentais e ONGs bem como incentivava a participação de portadores e seus familiares, o que estimulava o exercício da cidadania dessa população.

As ações desse Programa consistiam na participação e/ou organização de espaços coletivos de discussão e articulação política entre membros de ONGs e profissionais de saúde. Cada um dos espaços de discussão descritos a seguir tinha um objetivo e uma amplitude específicos: I - grupo de ambulatórios de AIDS: tratava-se de uma reunião técnica mensal do Programa Municipal de AIDS com os profissionais a ela vinculados para discutir questões relativas à organização e realização das ações do Programa. Através da participação do GHIV nessas reuniões, em rodízio com outras ONGs da cidade, buscavase acompanhar as ações de assistência em
AIDS nos serviços especializados da cidade, levando as necessidades dos portadores e difundindo as informações aí obtidas; II - grupo de ONGs/AIDS de Ribeirão Preto: através da iniciativa do GHIV, criou-se esse grupo, que tinha por objetivo estimular a articulação entre as seis ONGs/AIDS da cidade e promover a organização desse movimento social e a representação efetiva dos interesses dos portadores. O GHIV se responsabilizava pela organização mensal dos encontros bem como pelo oferecimento do local para a realização dos mesmos; III - Fórum de OGs e ONGs/ AIDS da Região Norte/Nordeste do Estado de São Paulo e IV - Fórum Estadual de ONGs/ AIDS de São Paulo: através da participação nesses fóruns, o GHIV buscava a articulação com representantes regionais e estaduais de ONGS/AIDS, visando à solução das dificuldades enfrentadas pelos portadores e a participação da cidade nas decisões do movimento de AIDS do Estado. A representação do GHIV, nessas reuniões, se dava através de uma equipe de voluntários da instituição.

O trabalho do psicólogo nesse Programa (atuação 8 e 9) era realizado por meio de reuniões de acompanhamento dos voluntários responsáveis pelas atividades-fim do Programa, cuja função era promover a reflexão sobre as ações realizadas, a integração da equipe do núcleo e o planejamento das atividades. A atuação do psicólogo, nessas atividades, contribuiu para o fortalecimento do senso crítico dos voluntários da ONG, fazendo dessa organização uma fonte de ações do movimento social em saúde e questionando o lugar de prestador de serviço, muitas vezes depositado e assumido pelas ONGs. A atuação psicológica propunha-se como uma prática libertadora em um contexto, muitas vezes, de indiferença e desrespeito aos direitos das pessoas portadoras. Entre os principais conceitos e valores que orientavam a atuação do psicólogo nesse Programa, estavam o de participação social, o de integração entre os portadores e o de eqüidade. A participação social do portador no 
estabelecimento de políticas públicas a ele destinadas sempre representou uma das formas mais amadurecidas de enfrentamento da epidemia e consistia em um modo eficaz de promoção de um jeito de viver com HIV/ AIDS, pautado pela responsabilidade e esperança. A criação de espaços de participação social facilitava a integração entre os portadores e fazia as necessidades individuais serem entendidas em suas dimensões sociais mais amplas, que promoviam um senso de segurança e potência e faziam do HIV um desafio a ser enfrentado coletivamente. Esse processo de enfrentamento coletivo, de busca por melhores condições de atendimento, implicava uma negociação com diversos setores da sociedade, incluindo órgãos governamentais e representantes da sociedade civil organizada. A especificidade das necessidades dos portadores era enfatizada nas negociações sobre políticas públicas destinadas a essa população, e era sustentada pela idéia de eqüidade. Esta, em substituição à idéia de igualdade, propunha que, em uma gestão mais justa dos recursos disponíveis, se tratasse diferentemente os diferentes, ou seja, diferentes montantes de recursos para diferentes tipos de necessidades.

\section{O compromisso social do trabalho do psicólogo em ONGs/AIDS}

Através da apresentação das atividades desenvolvidas pelos psicólogos em uma ONG/ AIDS, pudemos ver que muitas atividades realizadas nesse contexto também o são em diversos outros locais tradicionais de inserção do psicólogo. Contudo, há algumas questões que se colocam sobre o fazer psicológico, dada a história de surgimento desse tipo de instituição, as tarefas e compromissos a que ela se propõe e a população atendida e seu sofrimento. Entre essas questões, destacamse aquelas relativas à ampliação da definição de prática profissional, à relação desta com a promoção da cidadania, ao lugar da pessoa portadora na relação com o psicólogo e à conseqüente transformação do papel profissional.

Analisando comparativamente as ações realizadas pelos psicólogos em dois núcleos de atividades do GHIV, é possível visualizar uma forma de atuação profissional sensível às necessidades do contexto institucional, que mescla formas de atendimento clínicas e institucionais/comunitárias.

De um lado, as ações promovidas pelo Núcleo de Atenção Psicossocial propõem um cuidado psicológico tal como proposto por modelos tradicionais de saúde mental, como psicoterapia individual de longo prazo e grupos de apoio psicológico. Contudo, as atividades do Programa de Socialização e de Adesão buscam responder às necessidades dos portadores em seu contexto social imediato, no interior de relações familiares, de amizade e profissionais. Longe de serem atividades de recreação, as ações de socialização reconhecem o caráter sistêmico do fenômeno saúde-doença, no qual, muitas vezes, as redes sociais são rompidas em decorrência da discriminação e o isolamento se torna uma dificuldade no enfrentamento da doença. Além disso, as ações de promoção da adesão que privilegiam a inserção institucional da relação profissional de saúde-cliente combatem a culpabilização deste último e fomentam melhor qualidade de vida para todos os envolvidos.

Por outro lado, as ações do Núcleo de Cidadania Ativa implicam uma atuação profissional que considera os portadores sujeitos de direitos e que devem ter condições de exercê-los. Nesse contexto, as relações entre política e Psicologia se estreitam. Tradicionalmente, na busca de se posicionar como uma ciência objetiva, a Psicologia deixa de contribuir com os desafios da prática da
A criação de espaços de participação social facilitava a integração entre os portadores e fazia as necessidades individuais serem entendidas em suas dimensões sociais mais amplas, que promoviam um senso de segurança e potência e faziam do HIV um desafio a ser enfrentado coletivamente. 
Psicologia como profissão. Assim, a busca de neutralidade e de objetividade tem servido como justificativa para o não envolvimento de muitos psicólogos com questões políticas e sociais.

Contudo, no contexto da AIDS, segundo Altman, "A principal lição sobre saúde pública da epidemia é que é impossível oferecer uma prevenção eficaz e serviços de assistência sem se envolver no desenvolvimento comunitário e, em conseqüência, nas formas de intervenção política" (1995, p. 78). Para esse autor, as técnicas psicológicas de mudança de comportamento são insuficientes se não estiverem associadas a ações políticas. A AIDS vem, assim, exigir que a Psicologia contribua politicamente para o seu enfrentamento. Essa tarefa se coloca de forma mais significativa para aqueles que trabalham no contexto das ONGs/AIDS.

Neste artigo, buscamos mostrar como a assessoria que os psicólogos podem dar para os trabalhos em grupo, para a integração e melhor relação entre os ativistas e mesmo o apoio às denúncias e a busca por serviços de melhor qualidade consistem em uma forma de promover o exercício da cidadania de todos os envolvidos, em consonância com as propostas de uma psicologia crítica da saúde, "Advocacy, lobbying e parcerias de solidariedade são veículos para pressionar os governos a agir em defesa das populações vulneráveis. Apesar de o sistema médico formal não ser o único meio para se ter saúde, ele é um recurso social que precisa ser distribuído igualitariamente entre todos. Nós consideramos esse tipo de trabalho político como parte integral do trabalho de psicólogos críticos da saúde e psicólogos comunitários" (Prilleltensky, Prilleltensky, 2003, p. 208).

Nesse sentido, faz-se importante, tal como fizemos detalhadamente neste artigo, explicitar os valores que orientam a atuação profissional e as implicações políticas e sociais dos mesmos. Há um olhar psicológico que, ao compreender o ser humano em sua integralidade, reconhece a inserção social do mesmo e promove ações que visem a sua integração com o meio em que vive e transformem-no coletivamente, para seu bemestar. O psicólogo contribui, assim, para a mobilização política dos portadores, ao abandonar o discurso da passividade do cliente, comum nas falas dos profissionais no contexto dos serviços de saúde pública.

Esse imbricamento entre a assistência e a intervenção política faz com que, no contexto de uma ONG/AIDS, a pessoa portadora do HIV possa, ao mesmo tempo, ser um cliente, acompanhante de um cliente e um voluntário da instituição. É necessário reconhecer a pessoa soropositiva em sua multiplicidade, o que combate a visão parcial e fragmentada do portador como apenas um doente que demanda cuidados profissionais e questiona a lógica que sustenta a relação entre um psicólogo-especialista-cuidador e um portadorcliente-cuidado. Precisamos transformar nosso olhar para reconhecer, no portador, um parceiro, pois as ONGs/AIDS são marcadas por uma política de identidade através da qual se busca a produção de novos sujeitos sociais (Terto Jr., 1999). É preciso estar aberto bem como promover espaços para que o portador passe "da condição de civilmente morto à de politicamente vivo" (Silva, 2002, p. 93).

Essas características institucionais e da população atendida exigem um profissional híbrido, que, através de uma atuação multifacetada, combine conhecimentos ora da Psicologia clínica, ora da Psicologia social da saúde/comunitária, ora da Psicologia organizacional e de todos os saberes que contribuam para o reconhecimento da especificidade desse trabalho e permitam uma atuação eficaz e comprometida.

No processo de combinação desses diversos saberes e práticas psicológicas, é necessário, ainda, que o profissional ressignifique $\mathrm{o}$ preceito ético de sua prática, ou seja, que este 
deixe de ser uma prescrição técnica e legal e passe a representar a busca da construção conjunta entre psicólogo e pessoa portadora do HIV para o bem viver de todos.

O trabalho de uma equipe de psicólogos em uma ONG/AIDS, muitas vezes, não se faz sem o enfrentamento de diversos desafios: vários profissionais são recém-formados, com pouca experiência, e exigem supervisão cuidadosa; muitos voluntários da ONG são oriundos das classes populares, com pouca formação escolar; outros voluntários são portadores do HIV, o que Ihes exige esforços contínuos de significação da própria doença e da vivida por aqueles atendidos pela instituição; a população atendida enfrenta dificuldades em vários setores da existência - sejam econômicas, sociais e psicológicas, que exigem respostas nem sempre imediatas ou mesmo possíveis nesse contexto institucional, e, finalmente, a luta permanente pela sustentabilidade financeira da instituição.

Este relato da contribuição da Psicologia na construção de uma ONG/AIDS serve como um convite aos psicólogos para que explicitem os valores implícitos de suas ações, proponham práticas sensíveis ao contexto de trabalho e da população atendida, reconheçam a multiplicidade de práticas psicológicas e reflitam sobre as implicações políticas dos diferentes tipos de fazer psicológico, que sempre promovem determinadas formas de vida.

Vemos, assim, que a contribuição da Psicologia pode ser significativa; contudo, devemos vencer o desafio de transformarmos nossas práticas profissionais, de revermos os "especialismos" no interior da Psicologia e de assumirmos o compromisso social de nossa profissão, ao explicitar, tal como em outras áreas, como a AIDS veio questionar práticas tradicionais e exigir alternativas na atenção em saúde.

Emerson F. Rasera Doutor em Psicologia, Universidade Federal de Uberlândia - Instituto de Psicologia

Carmem Lucia Graminha Issa Especialista em Psicologia clínica- Grupo Humanitário de Incentivo à Vida Universidade Federal de Uberlândia - Instituto de Psicologia, Av: Pará, 1720, bloco 2C CEP: 38400902 Uberlândia - MG. Email: emersonrasera@uol.com.br

Recebido 22/05/06 Reformulado 24/07/06 Aprovado 22/01/07

ALTMAN, D. Poder e Comunidade: Respostas Organizacionais e Culturais à AIDS. Rio de Janeiro: Relume Dumará, ABIA, IMS/UERJ, 1995.

BUCHALLA, C.; PAIVA, V. Da compreensão da vulnerabilidade social ao enfoque multidisciplinar. Revista de Saúde Pública, São Paulo, v.36, pp.117-119, 2002.

CAMARA, C. Articulações entre governo e sociedade civil: um diferencial na resposta brasileira à AIDS. Impulso, Piracicaba, v.32, pp.57-67, 2002.

CAMARA, C.; LIMA, R. M. Histórico das ONGs/AIDS e sua contribuição no campo das lutas sociais. In: Abong (org.). Direitos Humanos, Cidadania e AIDS. Campinas: Autores Associados, 2000, pp.29-74.

CATALAN, J. Psychological interventions in infection with the human immunodeficiency virus. British Journal of Psychiatry, Londres, v.167, pp.104-11, 1995.

DIMENSTEIN, M. D. B. O Psicólogo no Contexto do Sistema Único de Saúde (SUS): Perfil Profissional e Perspectivas de Atuação nas Unidades Básicas de Saúde (UBS). Tese de doutorado em Psiquiatria e Saúde Mental. Instituto de Psiquiatria da Universidade Federal do Rio de Janeiro, Rio de Janeiro, 1998.

DIMENSTEIN, M. O psicólogo e o compromisso social no contexto da saúde coletiva. Psicologia em Estudo, Maringá, v.6, n.2, pp.57-63, 2001.
IDAC/BANCO DE HORAS. Primeiro Simpósio Subjetividade e AIDS - o Livro. Rio de Janeiro: Idac, 2000.

PRILLELTENSKY, I.; PRILLELTENSKY, O. Towards a Critical Health Psychology. Journal of Health Psychology, Londres, v.8, pp.197-210, 2003.

SALDANHA, A.A.W.; FIGUEIREDO, M. A. C.; COUTINHO, M.P.L. Atendimento Psicossocial à AIDS. A busca pelas questões subjetivas. Jornal Brasileiro de Doenças Sexualmente Transmissíveis, Niterói, v.16, pp.84-91, 2004.

SILVA, L. Construção de identidade em um espaço comunicativo: a experiência do Grupo Pela Vidda. Impulso, Piracicaba, v.32, pp.91108, 2002.

SILVA, W. A., BUCHALA, C. M., PAIVA, V., LATORRES, M. R. D, O., STALL, R.; HEARST, N. Prevenção de doenças sexualmente transmissíveis e AIDS entre jogadores juniores. Revista de Saúde Pública, São Paulo, v.36, p.68-75, 2002.

SPINK, M. J. Psicologia Social e Saúde. Petrópolis: Vozes, 2003.

TERTO, Jr. V. Soropositividade e políticas de identidade no Brasil. In: Barbosa, R. M.; Parker, R. (orgs.). Sexualidades pelo Avesso. Rio de Janeiro: IMS/UERJ; São Paulo: Ed. 34, 1999, pp. 99-119.

ZEGANS, L. S., GERHARD A. L.; COATES T. J. Psychotherapies for the person with HIV disease. Psychiatric Clinics of North America, Amsterdã, v.17, pp.149-162, 1994.
Referências 